\title{
Riscos ao desenvolvimento fetal associados a depressão na gravidez: Uma breve revisão
}

\author{
Risks to fetal development associated with depression in pregnancy: A brief review \\ Riesgos para el desarrollo fetal asociados con la depresión en el embarazo: Una breve revisión
}

Recebido: 13/06/2021 | Revisado: 19/06/2021 | Aceito: 06/07/2021 | Publicado: 16/07/2021

\author{
Antonio Ycaro Rodrigues Lucena \\ ORCID: https://orcid.org/0000-0003-3768-2298 \\ Universidade CEUMA, Brasil \\ E-mail: ycarolucena2009@gmail.com \\ Kassyo Lenno Sousa Dantas \\ ORCID: https://orcid.org/0000-0001-8191-6923 \\ Universidade CEUMA, Brasil \\ E-mail: kassyolenno@outlook.com \\ Jusciellyson da Silva Nava \\ ORCID: https://orcid.org/0000-0002-4147-5577 \\ Universidade CEUMA, Brasil \\ E-mail: jusciellysonnava@gmail.com \\ Mário Henrique de Souza Gomes Feitosa \\ ORCID: https://orcid.org/0000-0003-2254-2321 \\ Universidade CEUMA, Brasil \\ E-mail: Marionfsu@hotmail.com \\ Francis Aiala de Araújo Ferreira \\ ORCID: https://orcid.org/0000-0001-7301-0638 \\ Universidade CEUMA, Brasil \\ E-mail: francisaiala@hotmail.com \\ Ísis Cristina de Sousa \\ ORCID: https://orcid.org/0000-0003-3178-5149 \\ Universidade CEUMA, Brasil \\ E-mail: isis_cris1@hotmail.com \\ Janildes Maria Silva Gomes \\ ORCID: https://orcid.org/0000-0003-3961-1733 \\ Universidade CEUMA, Brasil \\ E-mail: janildes.green@hotmail.com
}

\begin{abstract}
Resumo
Objetivo: Observar as repercussões causadas pela depressão durante o período gestacional, em um contexto embrionário, apontando as principais alterações físicas, hormonais e psíquicas causadas pela depressão durante e após a gravidez ao feto. Metodologia: Foi realizado um levantamento bibliográfico de 30 publicações originais relevantes sobre o tema, utilizando os descritores: Depressão; Desenvolvimento Fetal e Embrionário; Gestação; Feto, nas bases de dados Scielo, Google Scholar e PUBMED. Foram utilizados critérios de inclusão e exclusão dos artigos, totalizando 23 publicações utilizadas para a redação do texto. Resultados e Discussão: A depressão (DP) grave é uma doença que afeta cerca de 300 milhões de pessoas em todo o mundo e, durante a gravidez, há a presença de efeitos psicobiológicos e fisiológicos sobre o corpo e mente feminina. Essas alterações, juntamente com o processo de depressão levam a uma condição psicopatológica que afeta diretamente o desenvolvimento da gravidez, detendo a capacidade de provocar alterações no desenvolvimento fetal, associada a riscos elevados para abortos espontâneos, partos prematuros e baixo peso ao nascer, além disso, mesmo após o nascimento, pode haver a presença de problemas comportamentais e emocionais a longo prazo. Considerações Finais: A depressão não tratada durante o período gestacional é associada a riscos para mãe e feto, podendo levar a quadros de aborto espontâneo ou parto prematuro e comprometimento do desenvolvimento fetal e problemas durante a vida adulta. Ressaltamos ainda a necessidade da realização de estudos em modelos de in vivo e in vitro para a disseminação de dados relativamente seguros.
\end{abstract}

Palavras-chave: Depressão; Desenvolvimento fetal e embrionário; Gestação; Feto.

\section{Abstract}

Objective: To observe the repercussions caused by depression during the gestational period, in an embryonic context, pointing out the main physical, hormonal and psychological changes caused by depression during and after pregnancy with the fetus. Methodology: A bibliographic survey of 30 relevant original publications on the subject was carried out, using the descriptors: Depression; Fetal and Embryonic Development; Gestation; Fetus, in the Scielo, Google Scholar and PUBMED databases. Inclusion and exclusion criteria of articles were used, totaling 23 publications used 
for the writing of the text. Results and discussion: Severe depression (PD) is a disease that affects about 300 million people worldwide and, during pregnancy, there is the presence of psychobiological and physiological effects on the female body and mind. These changes, together with the depression process lead to a psychopathological condition that directly affects the development of pregnancy, detaining the ability to cause changes in fetal development, associated with high risks for miscarriages, premature births and low birth weight, in addition , even after birth, longterm behavioral and emotional problems may be present. Final Considerations: Untreated depression during the gestational period is associated with risks for the mother and fetus, which can lead to situations of miscarriage or premature birth and impairment of fetal development and problems during adulthood. We also emphasize the need to carry out studies in in vivo and in vitro models for the dissemination of relatively safe data.

Keywords: Depression; Fetal and embryonic development; Gestation; Fetus.

\section{Resumen}

Objetivo: Observar las repercusiones que produce la depresión durante el período gestacional, en un contexto embrionario, señalando los principales cambios físicos, hormonales y psicológicos provocados por la depresión durante y después del embarazo con el feto. Metodología: Se realizó un relevamiento bibliográfico de 30 publicaciones originales relevantes sobre el tema, utilizando los descriptores: Depresión; Desarrollo fetal y embrionario; Gestación; Feto, en las bases de datos Scielo, Google Scholar y PUBMED. Se utilizaron criterios de inclusión y exclusión de artículos, totalizando 23 publicaciones utilizadas para la redacción del texto. Resultados y Discusión: La depresión severa (EP) es una enfermedad que afecta a cerca de 300 millones de personas en todo el mundo y, durante el embarazo, se presentan efectos psicobiológicos y fisiológicos en el cuerpo y la mente femenina. Estos cambios, junto con el proceso depresivo conducen a una condición psicopatológica que afecta directamente el desarrollo del embarazo, deteniendo la capacidad de provocar cambios en el desarrollo fetal, asociados a altos riesgos de abortos espontáneos, partos prematuros y bajo peso al nacer, además, incluso después puede haber problemas de nacimiento, conductuales y emocionales a largo plazo. Consideraciones finales: La depresión no tratada durante el período gestacional se asocia con riesgos para la madre y el feto, que pueden conducir a situaciones de aborto espontáneo o parto prematuro y deterioro del desarrollo fetal y problemas durante la edad adulta. También destacamos la necesidad de realizar estudios en modelos in vivo e in vitro para la difusión de datos relativamente seguros.

Palabras clave: Depresión; Desarrollo fetal y embrionario; Gestación; Feto.

\section{Introdução}

A depressão (DP) é uma doença que afeta cerca de 300 milhões de pessoas em todo o mundo, é um transtorno mental comum e que pode ser tratado, no entanto é considerado uma das principais causas de deficiência em todo o mundo, sendo considerada como um problema de saúde pela Organização Mundial da Saúde (OMS), tornando-se um problema de saúde pública mundial e um importante fator de risco para o desenvolvimento de outras psicopatologias, sendo ainda considerada a principal causa do suicídio. Não há uma causa pré-estabelecida e, a interação de vários fatores complexos, como influencias genéticas, psicológicas, sociais, bioquímicas e familiares são determinantes destaques nas pesquisas epidemiológicas como contribuintes no desenvolvimento da doença (Sousa et al., 2021; Marcus, 2009). Humor depressivo, perda de prazer ou interesse, alterações psicomotoras, redução do sono e da concentração e perda de energia e peso apresentam-se como os principais sintomas relacionados (Vismari et al., 2008; Sousa et al., 2021).

Segundo a Classificação Internacional de Doenças a depressão pode ser classificada mediante sua apresentação clínica como leve, moderada ou grave, onde em todos os casos o paciente apresentará os sinais clássicos da DP (rebaixamento do humor, redução da energia e diminuição da atividade) (Silva, 2008). Atinge pessoas de todos as idades, gêneros e experiências, no entanto as mulheres são mais susceptíveis a desenvolver depressão do que homens, principalmente mulheres grávidas. Embora os estudos demonstrem resultados conflitantes, complicações obstétricas em mulheres com DP são bastantes evidentes na literatura médica, estando relacionadas com a elevação da mortalidade e morbidade quando não há o tratamento adequado (Sousa et al., 2021; Vismari et al., 2008).

A literatura científica mostra que o período gravídico-puerperal tente com maior frequência ao aparecimento de transtornos mentais na mulher, sendo a depressão o transtorno com maior frequência. A depressão frequentemente é uma condição crônica e recorrente e, portanto, podemos dizer que o histórico de depressão seja um fator para o surgimento da DP durante a gravidez. A prevalência da depressão em gestantes é aproximadamente de $15 \%$ em países desenvolvidos e $22 \%$ em 
países em desenvolvimento, sendo ainda associada a transtornos psiquiátricos, baixa renda e estado civil solteira (Moreira \& Sarriera, 2008).

Durante a gravidez, há a presença de efeitos psicobiológicos e fisiológicos sobre o corpo e mente feminina, diretamente ligados as alterações hormonais (como nos níveis de progesterona e estrógeno e pela supressão do eixo hipotálamo-hipofisário-ovariano), que levam as alterações necessárias para a formação de um ambiente gestacional. Pesquisadores acreditam que a DP não tratada em gestantes pode levar a complicações obstétricas associadas a patologias puerperais, afetando tanto a mãe quanto o feto (Vismari et al., 2008).

Essas alterações, juntamente com o processo de depressão levam a uma condição psicopatológica que, apesar de a nível subclínico, afeta diretamente o desenvolvimento da gravidez, detendo a capacidade de provocar alterações no desenvolvimento fetal que podem ser evidenciadas durante o período de gestação e no pós-parto, ou seja, possui potencial de afetar negativamente os resultados do parto e do bem-estar neonatal e, quando não tratada, pode persistir mesmo após o nascimento, levando ao aparecimento de consequências cognitivas, comportamentais e emocionais para os filhos, podendo afetar o crescimento fetal, o temperamento do bebê e também o comportamento na infância. Ainda assim, muitos médicos obstetras minimizam as consequências trazidas pela DP no bem-estar e saúde da mulher e filho (Poromaa et al., 2017).

Portanto, visto como um sério problema de saúde pública, este trabalho tem o objetivo de nortear sobre as repercussões causadas pela depressão durante o período gestacional em um contexto embrionário, apontando as principais alterações físicas, hormonais e psíquicas causadas pela depressão durante e após a gravidez ao feto.

\section{Metodologia}

O presente trabalho é uma revisão da literatura. Para isso, foi realizado uma pesquisa qualitativa com suporte metodológico baseado nos seguintes referenciais teóricos (Estrela, 2018; Ludke \& André, 2011), através do levantamento bibliográfico de 30 publicações originais relevantes sobre o tema, utilizando os descritores: Depressão; Desenvolvimento Fetal e Embrionário; Gestação; Feto. Os trabalhos foram pesquisados nas bases de dados Scielo, Google Scholar e PUBMED. Para a inclusão dos trabalhos, foram utilizados os seguintes critérios: Artigos de pesquisa pertinentes ao tema (independente da data) e postagens relevantes de 2007 até o ano de 2021. Para exclusão, observou-se a ausência de pesquisas in vitro e in vivo nos trabalhos e artigos que não abordem o tema da forma desejada com o objetivo do trabalho, totalizando 23 publicações utilizadas para a redação do texto.

\section{Resultados e Discussão}

\section{Depressão na gravidez}

A depressão pré-natal, assim chamada a depressão durante a gestação, se encontra em um espectro de doenças mentais perinatais, com grande capacidade de trazer graves problemas tanto para a mãe como para o feto. Quando não a o diagnóstico e um efetivo tratamento, as consequências tornam-se evidentes na gestação e no pós-parto. Ainda que haja tratamento farmacológico, as drogas utilizadas atualmente vêm sendo associadas a possíveis riscos ao desenvolvimento fetal. No entanto, ainda é pouco estudada, visto que as investigações se concentram no período pós-parto (Silva et al., 2016; Silva et al., 2020).

O tratamento antidepressivo é realizado mediante os aspectos biológicos, psicológicos e sociais do paciente. Não há grandes diferenças na eficácia terapêutica entre os antidepressivos, no entanto, o preço, efeitos colaterais, riscos de suicídio e tolerabilidade geram padrões que implicam na efetividade da droga em cada paciente, levando assim a uma conduta de terapêutica individualizada, visto que atualmente existem drogas que atuam por diferentes mecanismos de ação (Souza, 1999; Marcus, 2009). 


\section{Tratamento da depressão durante a gravidez}

Há dificuldades dos profissionais de saúde na identificação precoce dos sinais e sintomas em considerá-los como um possível transtorno psíquico, visto que ainda existe um grande estigma envolvendo a aceitação da DP por parte do paciente, família e sociedade. Diante do diagnóstico de DP no período gestacional, algumas práticas terapêuticas são disponibilizadas, como a farmacoterapia, a psicoterapia e a eletroconvulsoterapia. Dentre as opções farmacológicas, as mais ultilizadas são os Inibidores Seletivos de Recaptação da Serotonina (ISRS), por exemplo a sertralina e citalopram são os mais utilizados (Camacho et al., 2006).

Segundo Chisolm e Payne (2016), os ISRS são os antidepressivos mais estudados para o tratamento depressivo em gestantes, no entanto, de acordo com a dose possui a capacidade de adentrar a barreira placentária e consequentemente elevar os níveis de serotonina na placenta, causando redução do fluxo sanguíneo nos espações intervilosos da placenta em decorrência do potencial vasodilatador da serotonina, podendo afetar as trocas gasosas e de nutrientes entre mãe e feto, retardando assim o desenvolvimento fetal. No entanto, ainda se apresentam como os antidepressivos mais utilizados por apresentarem maior segurança e eficácia (Jordan et al., 2019).

\section{Depressão e o desenvolvimento fetal}

As consequências a curto prazo da depressão durante o período gestacional incluem certas dificuldades na realização de tarefas habituais básicas, como a procura pela assistência médica para a realização do pré-natal, uso de substancias químicas nocivas como álcool e tabaco, uma dieta desbalanceada e o risco de automutilação e suicídio (Yonkers et al., 2009; Stewart, 2011).

A DP não tratada (DPNT) em mulheres grávidas vem sendo associada a riscos elevados para abortos espontâneos, partos prematuros e baixo peso ao nascer, pesquisas laboratoriais revelam níveis elevados de cortisol nessas mulheres, refletindo diretamente no desenvolvimento fetal e o aumento da irritabilidade da mãe. Contudo, esses achados são de difícil interpretação em virtude das possíveis consequências da DPNT e das consequências do uso dos medicamentos psicotrópicos utilizados durante o tratamento. Também podem ser associados ao uso de drogas como álcool e tabaco, o que aponta a necessidade de estudos clínicos randomizados para estes casos (Stewart, 2011; Okeane \& Marshms, 2007).

Kurki et al, (2000) em estudos com 623 mulheres finlandesas nulíparas com baixo risco para pré-eclâmpsia (complicação gestacional caracterizada por pressão arterial elevada com o surgimento de proteinúria), constatou que ao engravidarem, 30\% dessas mulheres foram testadas com depressão e ansiedade, e destas foram observados proteinúria e préeclâmpsia em 4,5\%. Portanto, no estudo em questão a depressão foi associada a um risco aumentado de pré-eclâmpsia, assim como a ansiedade.

Vários estudos também sugerem a DP como um grande fator de risco para a ocorrência de aborto espontâneo. Pesquisas apontam que o estresse em conjunto com os hormônios associados a depressão (hormônio liberador de corticotrofina - CRH e hormônio adrenocorticotrófico - ACTH) possuem potencial estimulador sobre células T e mastócitos induzindo a liberação de citocinas pró-inflamatórias, levando a um desequilíbrio no ambiente gestacional, como a ativação desproporcional da cascata de coagulação e consequente vasculite, afetando a oxigenação com posterior isquemia placentária, levando ao aborto espontâneo (Arck et al., 2001; Sugiura et al., 2002).

Além disso, mesmo após o nascimento, pode haver a presença de problemas comportamentais e emocionais a longo prazo, como o apego reduzido entre mãe e filho, atraso no desenvolvimento cognitivo e emocional, irritabilidade e resposta aumentada ao estresse. Ashman et al, (2002) avaliaram os níveis de cortisol salivar em filhos de mães com e sem depressão. As amostras foram colhidas após a chegada no laboratório (com fator estressante) e em um dia rotineiro (sem fator estressante) fora do ambiente laboratorial. Foi observado que os filhos de mães com depressão demonstraram níveis basais de cortisol 
elevados em resposta ao estresse comparados aos filhos de mães sem depressão.

Maki et al, (2003) sugeriram uma associação entre criminalidade e filhos de mães com depressão não tratada, e além disso, outros estudos demonstram que esses filhos possuem maior propensão para o desenvolvimento de DP quando comparado a filhos de mães saudáveis, o que sugere uma suscetibilidade genética, além dos fatores ambientais

Com base nas informações aqui relatadas e na pouca literatura publicada sobre as repercussões causadas pela depressão durante o período gestacional em modelos experimentais in vivo ou in vitro, a seguir encontrasse uma tabela com as principais consequências gestacionais/fetais (Tabela 01).

Tabela 1. Resumo das repercussões causadas pela depressão pré-natal.

ALTERAÇÕES FISIOPATOLÓGICAS

\begin{tabular}{|c|c|}
\hline PRÉ-ECLÂMPSIA & KURKI T, et al., 2000. \\
ABORTO ESPONTÂNEO & ARCK PC, et al., 2001; SUGIURA OM, et al., 2002. \\
ALTERAÇÕES NOS NÍVEIS DE CORTISOL \\
PROPENSÃO AO DESENVOLVIMENTO DE DP \\
APÓS O NASCIMENTO \\
ALTERAÇÕES COGNITIVAS, \\
COMPORTAMENTAIS E EMOCIONAIS \\
REDUÇÃO DO CRESCIMENTO FETAL \\
PARTOS PREMATUROS E BAIXO PESO AO 2002. \\
NASCER & HAMMEN C e BRENNAN PA, 2003. \\
\hline
\end{tabular}

Fonte: Lucena et al. (2021).

\section{Cuidados pós-parto}

As alterações fisiopatológicas decorrentes da DP na gestação no recém-nascido requerem uma adaptação complexa tanto dos pais e ambiente familiar como também da equipe multidisciplinar de assistência em todas as esferas da saúde no ambiente hospitalar e domiciliar, visto que reflete diretamente nos aspectos biológicos, psicológicos e sociais. Vivenciar um possível parto prematuro e complicações pós-parto trazem desafios desconhecidos que exigem recursos efetivos que, muitas vezes necessitam de habilidades modeladas para determinada situação e paciente (Rocha \& Chow-castillo, 2020; Busatto et al., 2021).

\section{Considerações Finais}

Visto então, com base nas informações aqui relatadas a depressão não tratada durante o período gestacional é associada a riscos para mãe e feto, podendo levar a quadros de aborto espontâneo ou parto prematuro e comprometimento do desenvolvimento fetal, alterações hormonais, propensão da criança ao desenvolvimento DP, alterações cognitivas, comportamentais e emocionais a longo prazo para mãe e filho, comprometendo diretamente a qualidade de vida.

Ressaltamos ainda a necessidade da realização de estudos em modelos de in vivo e in vitro que demonstrem detalhadamente os danos fetais e maternos envolvendo a depressão no período gestacional, para a disseminação de dados relativamente seguros, servindo de base para estudos mais complexos como o desenvolvimento de fármacos e medidas terapêuticas mais efetivas. 


\section{Referências}

Arck, P. C., Rose, M., Hertwig, K., Hagen, E., Hildebrandt, M., \& Klapp, B. F. (2001). Stress and immune mediators in miscarriage. Human Reproduction, $16(7), 1505-1511$.

Ashman, S. B., Dawson, G., Panagiotides, H., Yamada, E., \& Wilkinson, C. W. (2002). Stress hormone levels of children of depressed mothers. Development and psychopathology, 14(2), 333-349.

Busatto, E., Diaz, C. M. G., Teixeira, D. A., de Olivera, P. P., Benedetti, F. J., \& Costenaro, R. G. S. (2021). Cuidados com o recém-nascido após alta hospitalar: orientações aos pais. Research, Society and Development, 10(2), e30610212541-e30610212541.

Camacho, R. S., Cantinelli, F. S., Ribeiro, C. S., Cantilino, A., Gonsales, B. K., Braguittoni, É., \& Rennó Jr, J. (2006). Transtornos psiquiátricos na gestação e no puerpério: classificação, diagnóstico e tratamento. Archives of Clinical Psychiatry (São Paulo), 33(2), 92-102.

de Jesus Silva, M. M., Peres Rocha Carvalho Leite, E., Alves Nogueira, D., \& Clapis, M. J. (2016). Depression in pregnancy. Prevalence and associated factors. Investigación y educación en enfermería, 34(2), 342-350.

de Sousa, T. J. D., de Faria, P. H. A., Dantas, K. L. S., Pinto, M. N. M., de Assis Malerba, G., Freitas, M. S., \& Figueiredo, S. (2021). Plantas medicinales como alternativa terapéutica para el trastorno Depresivo Mayor (DMD). Revista Eletrônica Acervo Saúde, 13(2), e5646-e5646.

Estrela, C. (2018). Metodologia científica: ciência, ensino, pesquisa. Artes Médicas.

Hammen, C., \& Brennan, P. A. (2003). Severity, chronicity, and timing of maternal depression and risk for adolescent offspring diagnoses in a community sample. Archives of general psychiatry, 60(3), 253-258.

Jordan, S., Davies, G. I., Thayer, D. S., Tucker, D., \& Humphreys, I. (2019). Antidepressant prescriptions, discontinuation, depression and perinatal outcomes, including breastfeeding: a population cohort analysis. PloS one, 14(11), e0225133.

Kurki, T., Hiilesmaa, V., Raitasalo, R., Mattila, H., \& Ylikorkala, O. (2000). Depression and anxiety in early pregnancy and risk for preeclampsia. Obstetrics \& Gynecology, 95(4), 487-490.

Ludke, M., \& André, M. E. (2011). Pesquisa em educação: abordagens qualitativas. Em Aberto, 5(31).

Mäki, P., Veijola, J., Räsänen, P., Joukamaa, M., Valonen, P., Jokelainen, J., \& Isohanni, M. (2003). Criminality in the offspring of antenatally depressed mothers: a 33-year follow-up of the Northern Finland 1966 Birth Cohort. Journal of Affective Disorders, 74(3), $273-278$.

Marcus, S. M. (2009). Depression during pregnancy: rates, risks and consequences. Journal of Population Therapeutics and Clinical Pharmacology, 16(1).

Moreira, M. C., \& Sarriera, J. C. (2008). Satisfação e composição da rede de apoio social a gestantes adolescentes. Psicologia em estudo, $13(4)$, 781-789.

O'Keane, V., \& Marsh, M. S. (2007). Depression during pregnancy. Bmj, 334(7601), 1003-1005.

Rocha, A. M., \& Chow-Castillo, L. A. (2020). Os benefícios do Método Mãe Canguru na UTI neonatal. Educandi \& Civitas, 3(1).

Silva, G. A. D. (2008). Estudo longitudinal sobre prevalência e fatores de risco para depressão pós-parto em mães de baixa renda (Doctoral dissertation, Universidade de São Paulo).

Silva, M. M. D. J., Lima, G. S., Monteiro, J. C. D. S., \& Clapis, M. J. (2020). Depressão na gravidez: fatores de risco associados à sua ocorrência. SMAD. Revista eletrônica saúde mental álcool e drogas, 16(1), 1-12.

Souza, F. G. D. M. (1999). Tratamento da depressão. Brazilian Journal of Psychiatry, 21, 18-23.

Stewart, D. E. (2011). Depression during pregnancy. New England Journal of Medicine, 365(17), 1605-1611.

Sugiura-Ogasawara, M., Furukawa, T. A., Nakano, Y., Hori, S., Aoki, K., \& Kitamura, T. (2002). Depression as a potential causal factor in subsequent miscarriage in recurrent spontaneous aborters. Human Reproduction, 17(10), 2580-2584.

Sundström Poromaa, I., Comasco, E., Georgakis, M. K., \& Skalkidou, A. (2017). Sex differences in depression during pregnancy and the postpartum period. Journal of neuroscience research, $95(1-2), 719-730$.

Vigod, S. N., Wilson, C. A., \& Howard, L. M. (2016). Depression in pregnancy. Bmj, 352.

Vismari, L., Alves, G. J., \& Palermo-Neto, J. (2008). Depressão, antidepressivos e sistema imune: um novo olhar sobre um velho problema. Archives of Clinical Psychiatry, 35(5), 196-204.

Yonkers, K. A., Wisner, K. L., Stewart, D. E., Oberlander, T. F., Dell, D. L., Stotland, N., \& Lockwood, C. (2009). The management of depression during pregnancy: a report from the American Psychiatric Association and the American College of Obstetricians and Gynecologists. General hospital psychiatry, 31(5), 403-413. 